\title{
The effect of gadolinium-based contrast-agents on automated brain atrophy measurements by FreeSurfer in patients with multiple sclerosis
}

\author{
Ingrid Anne Lie ${ }^{1}$ (1) Emma Kerklingh ${ }^{2} \cdot$ Kristin Wesnes $^{1,3,4} \cdot$ David R. van Nederpelt $^{2} \cdot$ Iman Brouwer $^{2} \cdot$ \\ Øivind Torkildsen ${ }^{1,3} \cdot$ Kjell-Morten Myhr $^{1,3} \cdot$ Frederik Barkhof $^{2,5} \cdot$ Lars B $^{1,6} \cdot$ Hugo Vrenken $^{2}$
}

Received: 11 June 2021 / Revised: 7 September 2021 / Accepted: 12 October 2021 / Published online: 3 January 2022

(C) The Author(s) 2021

\begin{abstract}
Objective To determine whether reliable brain atrophy measures can be obtained from post-contrast 3D T1-weighted images in patients with multiple sclerosis (MS) using FreeSurfer.

Methods Twenty-two patients with MS were included, in which 3D T1-weighted MR images were obtained during the same scanner visit, with the same acquisition protocol, before and after administration of gadolinium-based contrast agents (GBCAs). Two FreeSurfer versions (v.6.0.1 and v.7.1.1.) were applied to calculate grey matter (GM) and white matter (WM) volumes and global and regional cortical thickness. The consistency between measures obtained in pre- and post-contrast images was assessed by intra-class correlation coefficient (ICC), the difference was investigated by paired t-tests, and the mean percentage increase or decrease was calculated for total WM and GM matter volume, total deep GM and thalamus volume, and mean cortical thickness.

Results Good to excellent reliability was found between all investigated measures, with ICC ranging from 0.926 to 0.996 , all $p$ values $<0.001$. GM volumes and cortical thickness measurements were significantly higher in post-contrast images by 3.1 to $17.4 \%$, while total WM volume decreased significantly by $1.7 \%$ (all $p$ values $<0.001$ ).

Conclusion The consistency between values obtained from pre- and post-contrast images was excellent, suggesting it may be possible to extract reliable brain atrophy measurements from T1-weighted images acquired after administration of GBCAs, using FreeSurfer. However, absolute values were systematically different between pre- and post-contrast images, meaning that such images should not be compared directly. Potential systematic effects, possibly dependent on GBCA dose or the delay time after contrast injection, should be investigated.

Trial registration Clinical trials.gov. identifier: NCT00360906.

Key Points

- The influence of gadolinium-based contrast agents (GBCAs) on atrophy measurements is still largely unknown and challenges the use of a considerable source of historical and prospective real-world data.

- In 22 patients with multiple sclerosis, the consistency between brain atrophy measurements obtained from pre-and post-contrast images was excellent, suggesting it may be possible to extract reliable atrophy measurements in $T 1$-weighted images acquired after administration of GBCAs, using FreeSurfer.

- Absolute values were systematically different between pre-and post-contrast images, meaning that such images should not be compared directly, and measurements extracted from certain regions (e.g., the temporal pole) should be interpreted with caution.
\end{abstract}

Ingrid Anne Lie

Ingrid.lie@uib.noAuthors and Affiliations

1 Department of Clinical Medicine, University of Bergen, Bergen, Norway

2 Department of Radiology and Nuclear Medicine, MS Center Amsterdam, Amsterdam Neuroscience, Amsterdam UMC, Location VUmc, De Boelelaan 1118, 1081 HZ Amsterdam, The Netherlands
3 Neuro-SysMed, Department of Neurology, Haukeland University Hospital, Bergen, Norway

4 Department of Neurology, St. Olav's University Hospital, Trondheim, Norway

5 Institutes of Neurology and Healthcare Engineering, UCL London, London, UK

6 Norwegian Multiple Sclerosis Competence Centre, Department of Neurology, Haukeland University Hospital, Bergen, Norway 
Keywords Multiple sclerosis $\cdot$ Magnetic resonance imaging $\cdot$ Gadolinium $\cdot$ Atrophy $\cdot$ Grey matter

$\begin{array}{ll}\text { Abbreviations } \\ \text { BBB } & \text { Blood-brain barrier } \\ \text { CNR } & \text { Contrast-to-noise ratio } \\ \text { CR } & \text { Contrast ratio } \\ \text { EDSS } & \text { Expanded Disability Status Scale } \\ \text { FFE } & \text { Fast field echo. } \\ \text { FLAIR } & \text { Fluid-attenuated inversion recovery } \\ \text { FLIRT } & \text { FMRIB's Linear Image Registration Tool } \\ \text { FSL } & \text { FMRIB Software Library } \\ \text { GBCAs } & \text { Gadolinium-based contrast-agents } \\ \text { GM } & \text { Grey matter } \\ \text { ICC } & \text { Intra-class correlation coefficient } \\ \text { IQMs } & \text { Image quality metrics } \\ \text { LST } & \text { Lesion segmentation tool } \\ \text { mL } & \text { Millilitres } \\ \text { mm } & \text { Millimetre } \\ \text { MPRAGE } & \text { Magnetization-prepared rapid gradient-echo } \\ \text { MRI } & \text { Magnetic resonance imaging } \\ \text { MRIQC } & \text { MRI Quality Control Tool } \\ \text { ms } & \text { Millisecond } \\ \text { MS } & \text { Multiple sclerosis } \\ \text { RRMS } & \text { Relapsing-remitting MS } \\ \text { SD } & \text { Standard deviation } \\ \text { SNR } & \text { Signal-to-noise ratio } \\ \text { SPMS } & \text { Secondary progressive multiple sclerosis } \\ \text { SPSS } & \text { Statistical Product and Service Solutions } \\ \text { T } & \text { Tesla } \\ \text { T1 } & \text { Inversion time } \\ \text { TE } & \text { Echo time } \\ \text { TR } & \text { Repetition time } \\ \text { WM } & \text { White matter } \\ & \end{array}$

\section{Introduction}

Grey matter (GM) atrophy measured on MRI in persons with multiple sclerosis (MS) reflects irreversible neuroaxonal loss and neurodegenerative changes in the CNS [1]. The degree of GM atrophy has been shown to consistently correlate with physical $[2,3]$ and cognitive [4] disability, and is regarded as a promising neurodegenerative biomarker. Furthermore, as the demand for neuroprotective interventions increases, GM atrophy is an easily available outcome measure [5-7].

There are a number of available methods and software to measure GM atrophy. Although FreeSurfer requires substantial processing time, making it less suitable for clinical practice, it is one of the most commonly used automated methods in research, especially for cortical parcellation and thickness estimation. FreeSurfer is publicly available and widely validated [8-12], and in the body of literature on
GM atrophy in MS, many key papers have used FreeSurfer [13-15].

Due to the high tissue contrast [16-18] in unenhanced three-dimensional (3D) T1-weighted images, this image type is commonly used by brain segmentation software and required by FreeSurfer, as well as other software with similar purposes. However, unenhanced 3D T1-weighted images are not mandatory in suggested standardised brain MRI protocols for MS [19] and may not be routinely included. Instead, post-contrast T1-weighted images are often prioritised, especially in clinical settings. In case of ongoing inflammation, the intravenously administered contrast agent leaks into the brain parenchyma in locations where the blood-brain barrier (BBB) is disrupted [20]. These postcontrast images are valuable both in baseline and follow-up examinations, as they can unequivocally detect lesions with active inflammation [19].

The contrast agent used is almost universally gadolinium-based, consisting of a central paramagnetic $\mathrm{Gd}^{3+}$ ion chelated to a carrier molecule to prevent the toxicity of free $\mathrm{Gd}^{3+}$, while still maintaining its paramagnetic properties. Gadolinium-based contrast agents (GBCAs) shorten both the longitudinal (T1) and transverse (T2) relaxation times [21], leaving areas in which GBCAs accumulate as bright or hyperintense compared to surrounding tissue on T1-weighted images.

The use of GBCAs has increased over the last three decades [22], making up a considerable source of historical and prospective real-world data. However, the value of such data for brain atrophy measurements depends on our ability to correctly interpret the data in automated image analyses. The influence of GBCAs on atrophy measurements is still largely unknown and has previously been investigated in only a few studies using different image analysis techniques [23, 24]. In this study, our aim is to validate the use of post-contrast T1-weighted images for volume and cortical thickness measurements and to provide guidelines on how to interpret results from clinically relevant and commonly considered measures. To do so, total WM and GM volume, total deep GM and thalamus volume, and mean cortical thickness measures were obtained in pre- and post-contrast images by FreeSurfer and compared.

\section{Materials and methods}

\section{Participants}

The patients included in this study participated in a 10-year follow-up visit following a multi-centre, randomised, 
placebo-controlled trial of $\omega-3$ fatty acids in MS (the OFAMS-study), which has previously been described in detail [25]. A total of 85 of the 92 persons with relapsing-remitting MS (RRMS) [26] originally enrolled in the OFAMS-study participated in the 10-year follow-up visit and underwent clinical, biochemical, and radiological examinations at their local study site.

The study was approved by the Regional Committee for Medical and Health Research Ethics in Western Norway Regional Health Authority (clinical trials.gov, identifier: NCT00360906). All participants gave their written informed consent.

\section{MRI data and analysis}

\section{MRI data acquisition}

Imaging at the 10-year follow-up visit was performed at the different study sites, on a 3-Tesla (T) MRI scanner if available, alternatively using a $1.5 \mathrm{~T}$ MRI scanner, with a standard head coil. The acquisition included a postcontrast sagittal 3D T1-weighted sequence; acquisition details across sites are provided in Table 1. Furthermore, a sagittal T2-weighted 3D fluid-attenuated inversion recovery (FLAIR) sequence was acquired according to locally optimised protocols. The full MRI protocol provided to the study sites is available in eAppendix 1 . The study sites were encouraged to include the same 3D T1-weighted sequence before contrast-agent administration, if possible. For the present study, only the subset of the participants who underwent 3D T1-weighted MR imaging both before and after injection of GBCAs, during the same scanner visit, and with the exact same acquisition protocol, was included.

\section{MRI data processing}

Lesion segmentation and lesion filling Lesion segmentation was done on FLAIR images using lesion segmentation tool (LST) (version 2.0.15; http://applied-statistics. de/lst.html) [27]. The lesion probability map in FLAIR space was brought to T1-weighted space by FLIRT linear registration of the FLAIR image to the T1 image, using 7 degrees of freedom, correlation ratio as the cost function, and trilinear interpolation. Afterwards, a threshold of 0.1 was used to binarise the lesion probability map. To optimize the lesion filling, gadolinium-enhancing regions (both lesions and other regions) were first removed, by applying an upper-intensity threshold at the $98^{\text {th }}$ percentile. Next, the FMRIB Software Library (FSL) (version 5.0.10; http://www. fmrib.ox.ac.uk/fsl) was used to fill in lesional voxels in the T1-weighted images using the lesion_filling tool [28], and these filled lesions were pasted into the original post-contrast 3D T1-weighted images.

Morphological reconstruction Cortical reconstruction and parcellation for cortical volume and thickness measurement and subcortical segmentation were performed with FreeSurfer, a freely available software package for academic use, available through online download (http://surfer.nmr.mgh.harvard.edu/). The findings presented here were obtained using FreeSurfer version 7.1.1; highly comparable findings obtained using FreeSurfer version 6.0.1 are presented in Table e1. The technical details of FreeSurfer procedures have been previously described $[29,30]$ and briefly summarised in eAppendix 2 .

Quality control was performed by visual inspection, and any segmentation errors were recorded for each patient. In cases where only specific anatomical regions were incorrectly

Table 1 Details on MRI acquisition per protocol

\begin{tabular}{|c|c|c|c|c|c|c|c|}
\hline $\begin{array}{l}\text { Protocol (number } \\
\text { of patients) }\end{array}$ & $1(3)$ & $2(3)$ & $3(3)$ & $4(3)$ & $5(5)$ & $6(2)$ & $7(3)$ \\
\hline Scanner & Siemens Aera & Siemens Skyra & Siemens Avanto & Siemens Aera & Philips Achieva & Siemens Prisma & Philips Achieva \\
\hline Field strength & $1.5 \mathrm{~T}$ & $3 \mathrm{~T}$ & $1.5 \mathrm{~T}$ & $1.5 \mathrm{~T}$ & $1.5 \mathrm{~T}$ & $3 \mathrm{~T}$ & $1.5 \mathrm{~T}$ \\
\hline 3DT1 sequences & MPRAGE & MPRAGE & MPRAGE & MPRAGE & FFE & MPRAGE & FFE \\
\hline $\mathrm{TR}(\mathrm{ms})$ & 1940 & 2300 & 2200 & 2200 & 7.6 & 1800 & 7.1 \\
\hline $\mathrm{TE}(\mathrm{ms})$ & 2.69 & 2.32 & 2.82 & 2.67 & 3.75 & 2.28 & 2.2 \\
\hline $\mathrm{TI}(\mathrm{ms})$ & 976 & 900 & 900 & 900 & & 900 & \\
\hline Flip angle $\left(^{\circ}\right)$ & 8 & 8 & 8 & 8 & 8 & 8 & 8 \\
\hline Voxel size & $1.00 \times 0.98 \times 0.98$ & $0.9 \times 0.94 \times 0.94$ & $1.00 \times 0.98 \times 0.98$ & $1.00 \times 0.98 \times 0.98$ & $1.00 \times 0.98 \times 0.98$ & $1.00 \times 0.50 \times 0.50$ & $\begin{array}{l}1.00 \times 1.00 \\
\times 1.00\end{array}$ \\
\hline Head receiver coil & Unknown & Unknown & HE1-4 & HE1-4 & SENSE-head-8 & Unknown & SENSE-head-8 \\
\hline GBCA & $\begin{array}{l}\text { Gadoterate meglu- } \\
\text { mine }\end{array}$ & $\begin{array}{l}\text { Gadoterate meg- } \\
\text { lumine }\end{array}$ & $\begin{array}{l}\text { Gadoterate meg- } \\
\text { lumine }\end{array}$ & Gadoteridol & Unknown & $\begin{array}{l}\text { Gadoterate meglu- } \\
\text { mine }\end{array}$ & Unknown \\
\hline
\end{tabular}

Abbreviations: $T R$, repetition time; $T E$, echo time; $T I$, inversion time; $m s$, millisecond; $m m$, millimetre; $M P R A G E$, magnetization-prepared rapid gradient-echo; $F F E$, fast field echo; $G B C A$, gadolinium-based contrast agent 
segmented, we chose to not apply any corrections for these errors in our analyses.

The Desikan-Killiany atlas [31] was used to extract cortical thickness measures (mean cortical thickness, left and right hemisphere) and to study regional differences in cortical thickness between pre- and post-contrast images, across subjects, by creating a heat map. Furthermore, total cerebral GM and WM volume and total deep GM and thalamus volume (left and right hemisphere) were obtained.

MRI quality control tool To investigate potential root causes of any observed segmentation differences, both pre- and post-contrast $\mathrm{T} 1$-weighted images were analysed using the MRI Quality Control Tool (MRIQC) [32]. MRIQC is an open-source software and extracts no-reference image quality metrics (IQMs) from structural and functional MRI data [32]. Using a segmentation into GM, WM, and CSF by FSL-FAST [33], MRIQC calculates tissue-specific signalto-noise ratio (SNR) values as well as the contrast-to-noise ratio (CNR) between GM and WM. Additionally, based on these values obtained from MRIQC, the contrast ratio (CR) between white and grey matter was also calculated.

\section{Statistical analysis}

Statistical analyses were performed using the Statistical Product and Service Solutions (SPSS) for macOS (Version 25; SPSS). Data were visually and statistically examined using the Kolmogorov-Smirnov test for normality. To assess the agreement between volume and thickness measurements obtained before and after GBCA administration, the intra-class correlation coefficient (ICC) was determined, based on a mean rating $(k=2)$, consistency, two-way mixed model. Scatterplots were created to visualise the agreement. To assess whether any systematic differences in structural measurements or IQMs were present between pre- and postcontrast measurements, paired t-tests were performed. Furthermore, boxplots were made to illustrate any differences, and Bland-Altman plots were created to identify fixed or proportional bias [34]. As an exploratory analysis, paired $\mathrm{t}$-tests were used to investigate a possible systematic difference between field strengths (1.5 and $3 \mathrm{~T})$.

\section{Results}

Pre- and post-contrast T1-weighted images were obtained with the exact same acquisition protocol in a total of 23 patients. One patient was excluded due to a large image artifact, causing segmentation errors. Table 2 provides an overview of the demographic and clinical characteristics of the patient group.
Table 2 Demographic and clinical characteristics

\begin{tabular}{ll}
\hline Age in years, mean (SD) & $50.5(7.83)$ \\
\hline Sex, female, $N(\%)$ & $15(68.2 \%)$ \\
Disease duration, mean in years & $13.8(3) / 13(12-25)$ \\
$\quad$ (SD) / median (range) & \\
EDSS, mean (SD) / median (range) & $2.9(1.2) / 2.5(1-6)$ \\
Disease phenotype (N) & RRMS (21), SPMS (1) \\
Study site (number of patients) & Site 1 (3), Site 2 (3), Site 3 (3), \\
& Site 4 (8 (2 scanners)), Site 5 \\
& $(2)$, Site 6 (3) \\
\hline
\end{tabular}

Abbreviations: $S D$, standard deviation; EDSS, Expanded Disability Status Scale; RRMS, relapsing-remitting multiple sclerosis; SPMS, secondary progressive multiple sclerosis

\section{Quality control of FreeSurfer segmentations}

All 22 pairs of pre- and post-contrast T1-weighted images finished the fully automated FreeSurfer pipeline (i.e., no hard failures). The most common soft failures (i.e., failures that do not disrupt the pipeline, but may need modification) are summarised in Table 3.

\section{Volume and cortical thickness measurements before and after administration of GBCAs}

The mean values of MRI measurements obtained before and after GBCAs are summarised in Table 4 and Fig. 4. Briefly, a mean increase in GM volumes and cortical thickness measures were observed in post-contrast images, while a mean decrease was observed in total WM volume. The results of the exploratory analysis subdivided according to field strength are presented in Table e2, showing no clear systematic differences between field strengths.

\section{Consistency of measurements obtained before and after administration of GBCAs}

A high degree of reliability was found between the measurements obtained pre- and post-contrast, for all volumes and cortical thickness measures assessed. All ICC values (Table 4) were above 0.92 , with the lowest values in the thalami, and above 0.96 for all larger structures, all $p$ values $<0.001$. The consistency between the measurements is demonstrated in Fig. 5.

\section{Difference in measurements before and after administration of GBCAs}

GM volumes and mean cortical thickness were significantly higher after administration of GBCAs, in all investigated structures (Table 4, Figs. 4 and 5). 
Table 3 Summary of the most common soft failures

\begin{tabular}{lc}
\hline Description & Frequency \\
\hline $\begin{array}{l}\text { The pial surface (representing the border between cortical GM and } \\
\text { CSF) or the border of segmented deep GM structures, expanding } \\
\text { into extraparenchymal tissue, including components of dura or blood } \\
\text { vessels as part of the cortex or deep GM structures (Figs. 1b and 2) }\end{array}$ & $\begin{array}{c}\text { Found in all scans, both pre- and post-contrast, but more frequently and } \\
\text { to a more severe degree in post-contrast images }\end{array}$ \\
$\begin{array}{l}\text { The pial surface failing to follow the white surface, causing "looping" } \\
\text { errors (Fig. 1a) and subsequent incorrect enlargement of the cortical } \\
\text { volume and thickness }\end{array}$ & $\begin{array}{c}\text { Found in all scans, both pre- and post-contrast, but more frequently and } \\
\text { to a more severe degree in post-contrast images }\end{array}$ \\
$\begin{array}{c}\text { The constructed surface border between WM and GM (the white sur- } \\
\text { face) failing to follow the intensity gradient correctly in the temporal } \\
\text { poles, resulting in a suboptimal segmentation (Fig. 3) }\end{array}$ & $\begin{array}{c}\text { Found to a moderate degree in two post-contrast images, and to a minor } \\
\text { degree in a total of eight patients, in the post-contrast image in all } \\
\text { eight, and in the pre-contrast image in three of those eight }\end{array}$ \\
\hline
\end{tabular}

Abbreviations: $G M$, grey matter; $C S F$, cerebrospinal fluid; $W M$, white matter

Fig. 1 Post-contrast T1-weighted MRI, showing the border between WM and GM (white surface) (yellow), and the border between GM and CSF (pial surface) (red). a Axial slice showing a moderate pial surface "looping error" (white arrow). b Sagittal slice showing a typical skull stripping failure; a moderate error of the pial surface expanding into the dura and the sagittal sinus (white arrow)

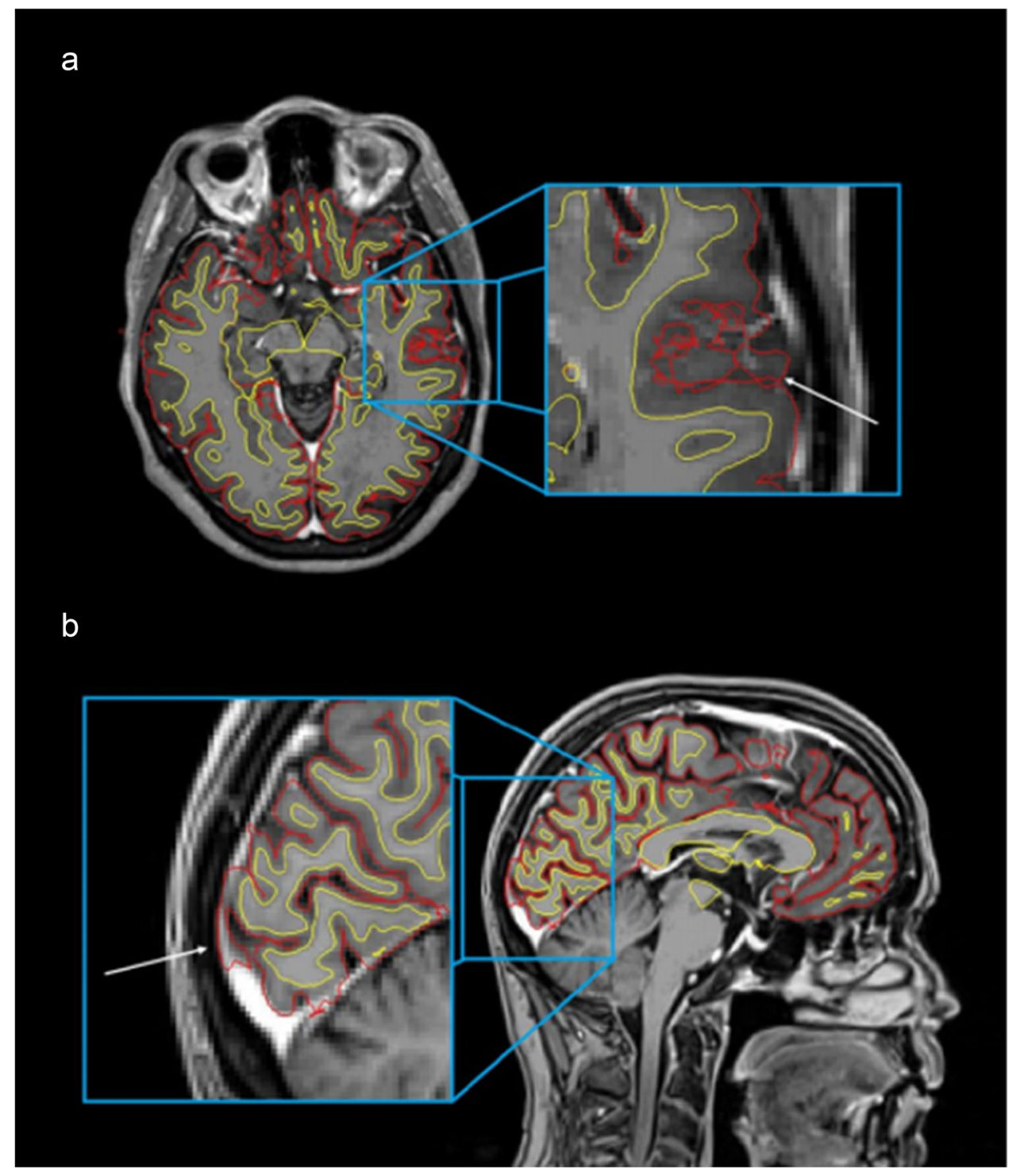




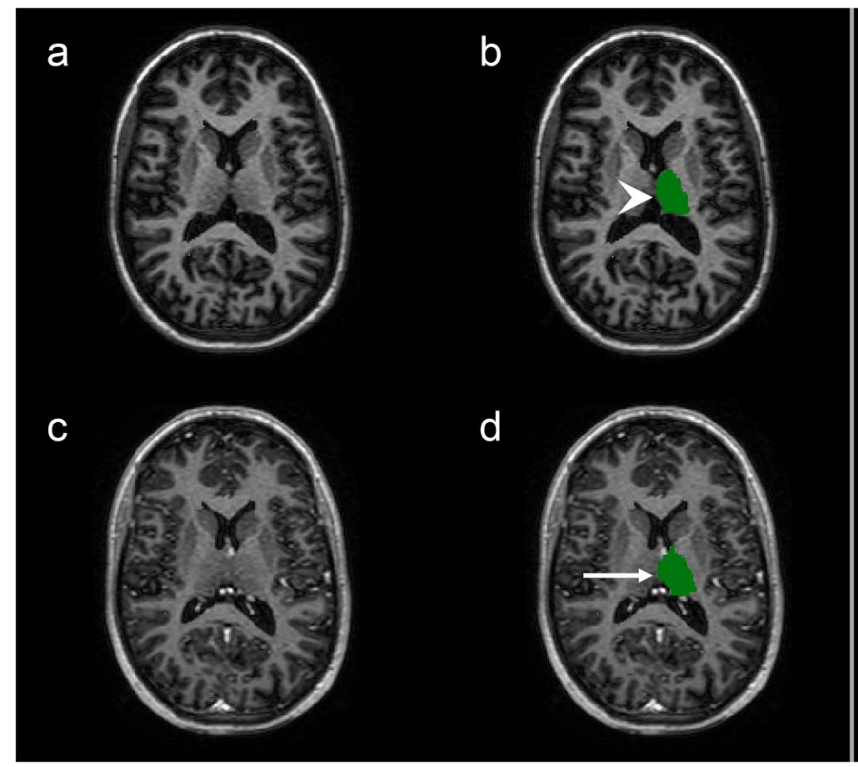

Fig. 2 T1-weighted MRI, showing the segmentation of the left Thalamus in pre- and post-contrast images, in two different patients (subject E3 (a-d) and subject $\mathrm{C} 1(\mathbf{e}-\mathbf{h}))$. a-d Axial slices demonstrating the typical quality of thalamus segmentations. In post-contrast images (c-d), the medial border of the left Thalamus is slightly overestimated (arrow) compared to pre-contrast images (arrowhead) (a-b), most likely due to hyperintense signal from extraparenchymal struc-

Figure 6 shows heatmaps visualising the difference in cortical thickness between pre- and post-contrast images, demonstrating the general increase in thickness measured in post-contrast T1-weighted images. However, in a few exceptions, most prominently the temporal pole, the parahippocampal, and the entorhinal gyrus in the temporal lobe, cortical thickness decreased.

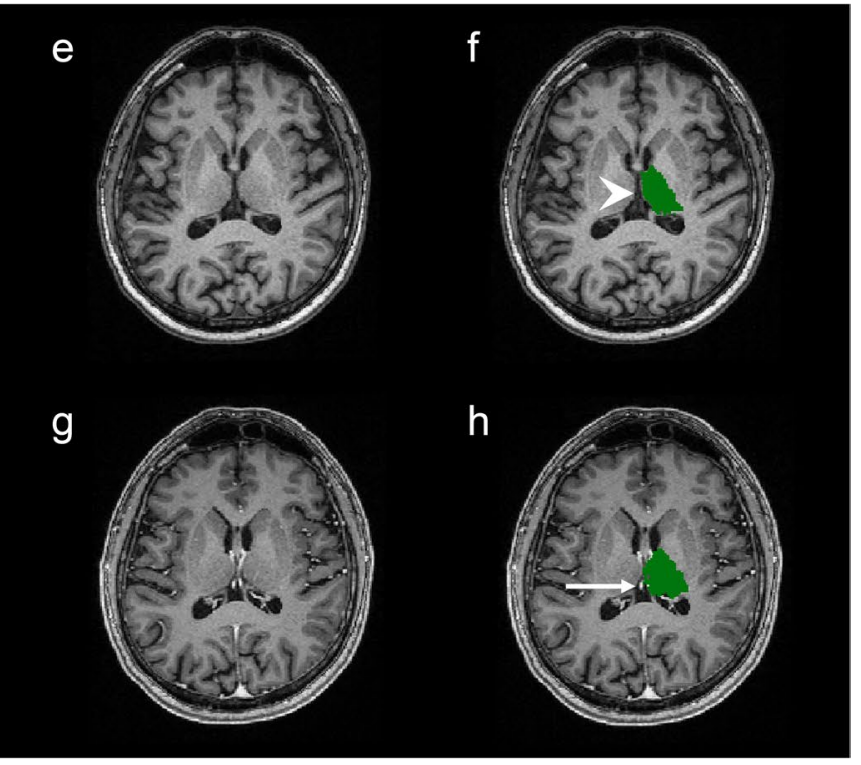

tures in the midline. $\mathbf{e}-\mathbf{h}$ Axial slices demonstrating a more severe overestimation of the medial border of the left Thalamus (arrow) in post-contrast images $(\mathbf{g}-\mathbf{h})$ compared to pre-contrast images (arrow head) (e-f). Again, the segmentation of the medial border is overestimated due to inclusion of extraparenchymal hyperintense structures, in this case, the internal cerebral vein)

While GM volumes and cortical thickness measurements were higher after administration of GBCAs, total WM volume was significantly lower. Figure e1 in the supplementary material shows the constructed Bland-Altman plots, revealing systematic differences, but no proportional bias.

IQMs are reported in Table 5. The CNR was not significantly different between pre- and post-contrast images.
Fig. 3 Pre-contrast $(\mathbf{a}-\mathbf{c})$ and post-contrast (d-f) T1-weighted images obtained from the same patient (subject A3) in the same MRI session. $\mathbf{b}$ and $\mathbf{e}$ show the white surface, which is the border between white and grey matter as automatically constructed by FreeSurfer (yellow). $\mathbf{c}$ and f show the pial surface, which is similarly the automatically constructed border between grey matter and cerebrospinal fluid (red), derived from the white surface. The figure demonstrates a typical failure of moderate degree, where the white surface fails to include parts of the temporal poles in the postcontrast image (e) (arrow), with subsequent mistakes in the pial surface (f) (arrowhead)

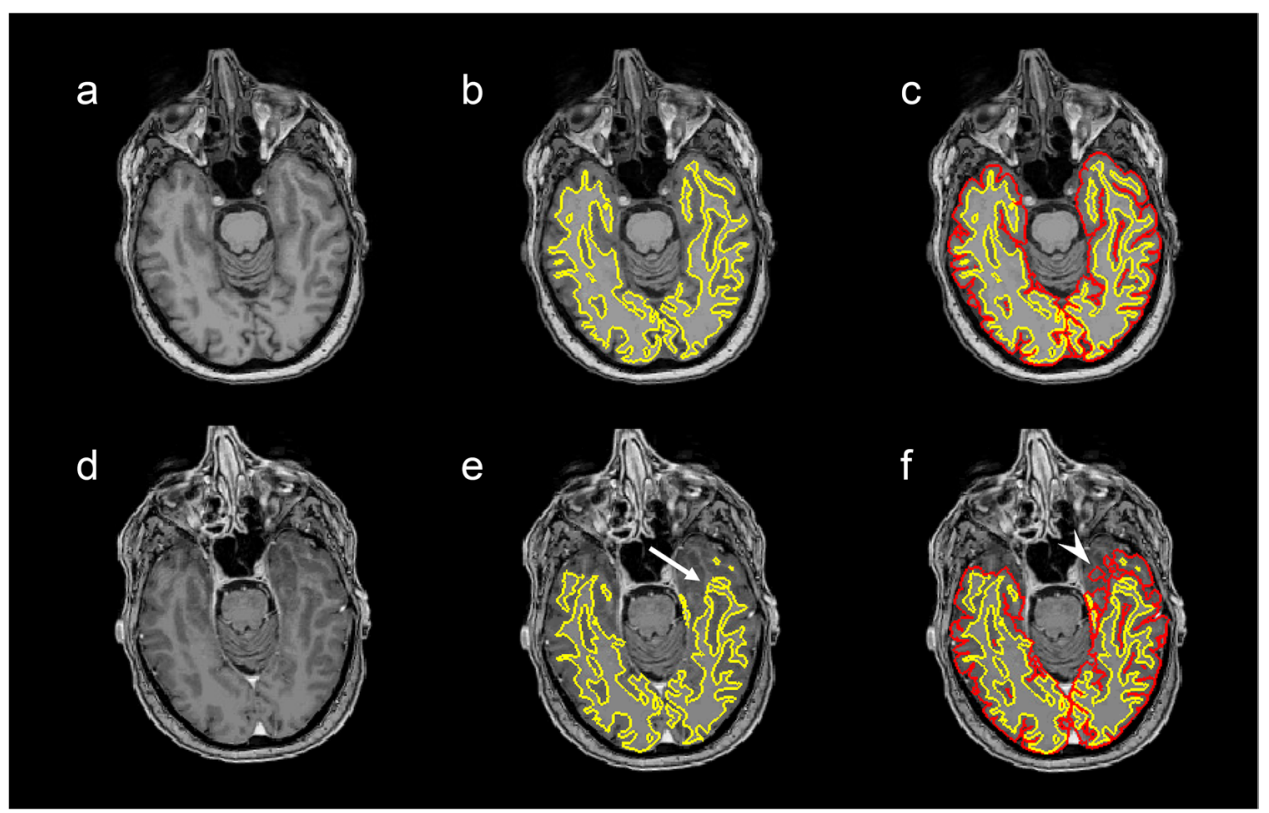


Table 4 MRI measurement values

\begin{tabular}{|c|c|c|c|c|c|}
\hline MRI measure & $\begin{array}{l}\text { Mean value } \\
\text { pre-contrast } \\
\text { (SD) }\end{array}$ & $\begin{array}{l}\text { Mean value } \\
\text { post-contrast } \\
\text { (SD) }\end{array}$ & Mean difference $^{\mathrm{a}}(\mathrm{SD})$ & $\begin{array}{l}\text { Percent increase/ } \\
\text { decrease (SD) }\end{array}$ & ICC (95\% confidence interval) \\
\hline Total grey matter volume (mL) & $602.53(62.42)$ & $620.33(59.97)$ & $17.80(16.20)^{* *}$ & $+3.06(2.79) \%$ & $0.982(0.957-0.993)$ \\
\hline Total white matter volume (mL) & $457.06(63.05)$ & $448.70(59.25)$ & $-8.36(7.35)^{* *}$ & $-1.74(1.48) \%$ & $0.996(0.991,0.998)$ \\
\hline $\begin{array}{l}\text { Total deep grey matter volume } \\
(\mathrm{mL})\end{array}$ & $51.57(5.90)$ & $54.73(5.61)$ & $3.16(2.04)^{* *}$ & $+6.33(4.43) \%$ & $0.968(0.922-0.987)$ \\
\hline Left thalamus volume (mL) & $6.47(0.93)$ & $7.58(1.09)$ & $1.10(0.48)^{* *}$ & $+17.39(8.46) \%$ & $0.940(0.855-0.975)$ \\
\hline Right thalamus volume (mL) & $6.37(0.99)$ & $7.12(0.92)$ & $0.75(0.50)^{* *}$ & $+12.52(9.04) \%$ & $0.926(0.823-0.969)$ \\
\hline $\begin{array}{l}\text { Mean cortical thickness left hemi- } \\
\text { sphere }(\mathrm{mm})\end{array}$ & $2.32(0.16)$ & $2.49(0.15)$ & $0.17(0.06)^{* *}$ & $+7.38(2.78) \%$ & $0.964(0.913,0.985)$ \\
\hline $\begin{array}{l}\text { Mean cortical thickness right } \\
\text { hemisphere }(\mathrm{mm})\end{array}$ & $2.33(0.14)$ & $2.49(0.14)$ & $0.16(0.05)^{* *}$ & $+7.13(2.61) \%$ & $0.961(0.906,0.984)$ \\
\hline
\end{tabular}

Abbreviations: $S D$, standard deviation; $I C C$, intra-class correlation coefficient for consistency; $m L$, millilitres; $m m$, millimetre

${ }^{\text {a }}$ Paired t-test

${ }^{* * *} p<0.001$

Tissue-specific SNRs were significantly lower in post-contrast images, for both GM $(p<0.01)$ and WM $(p<0.0001)$. The CR between WM and GM was significantly higher in post-contrast images $(p<0.006)$.

\section{Discussion}

Our results demonstrate that using FreeSurfer, reliable GM volume- and cortical thickness measurements may be a

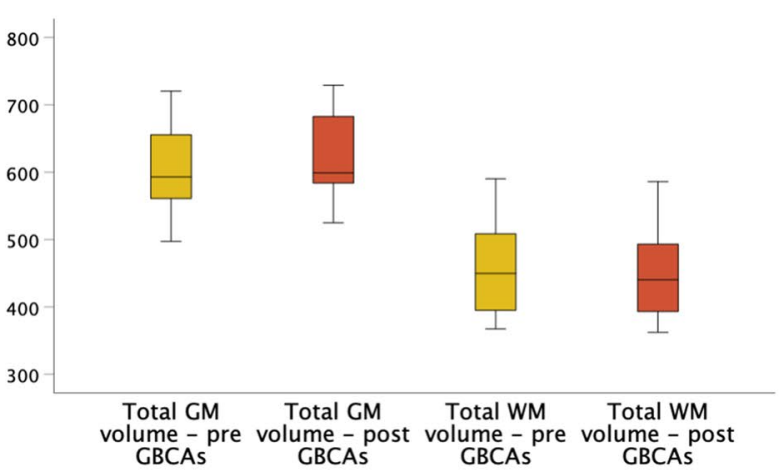

C

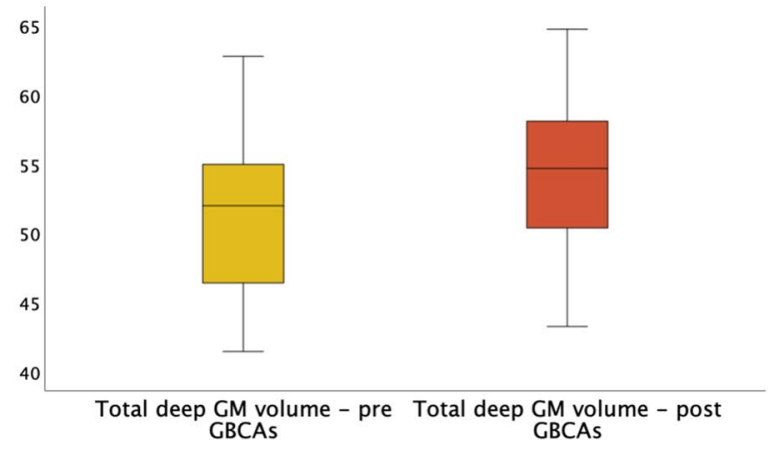

b
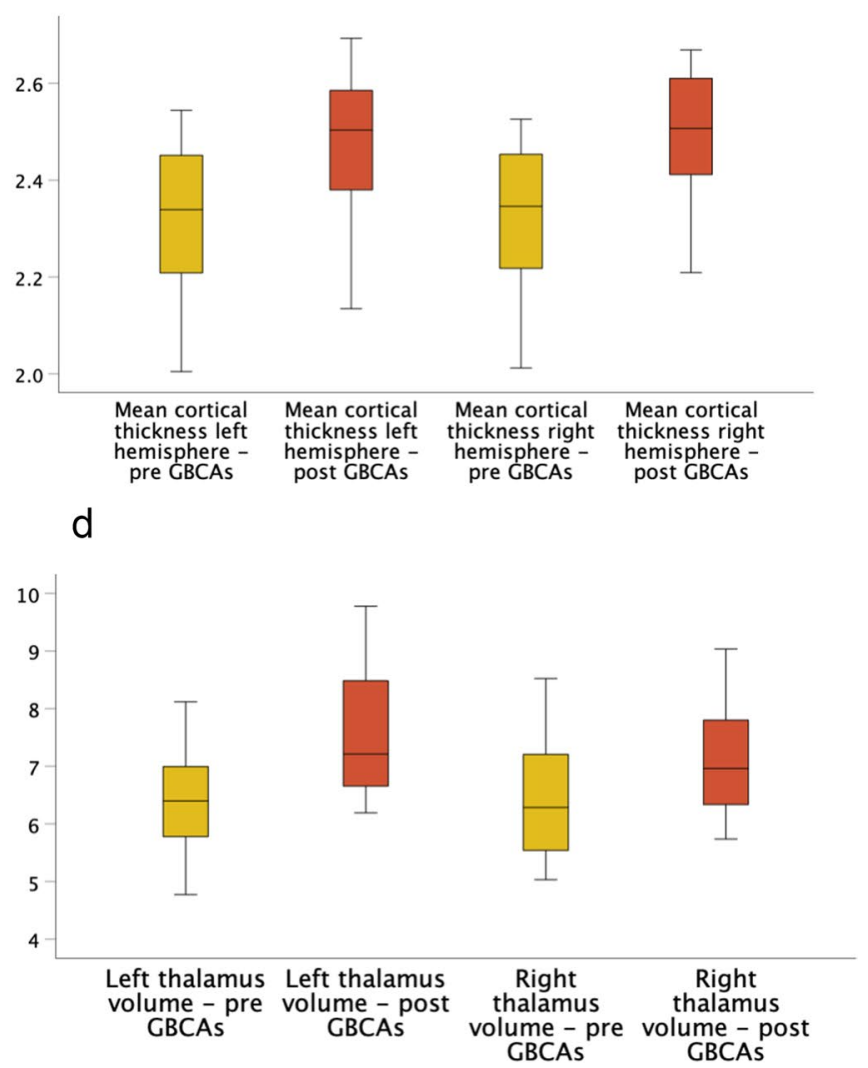

Fig. 4 Boxplots of MRI measurements obtained before (yellow) and after (red) GBCA administration, in $\mathrm{mL}(\mathbf{a}, \mathbf{c}, \mathbf{d})$ and $\mathrm{mm}(\mathbf{b})$ 

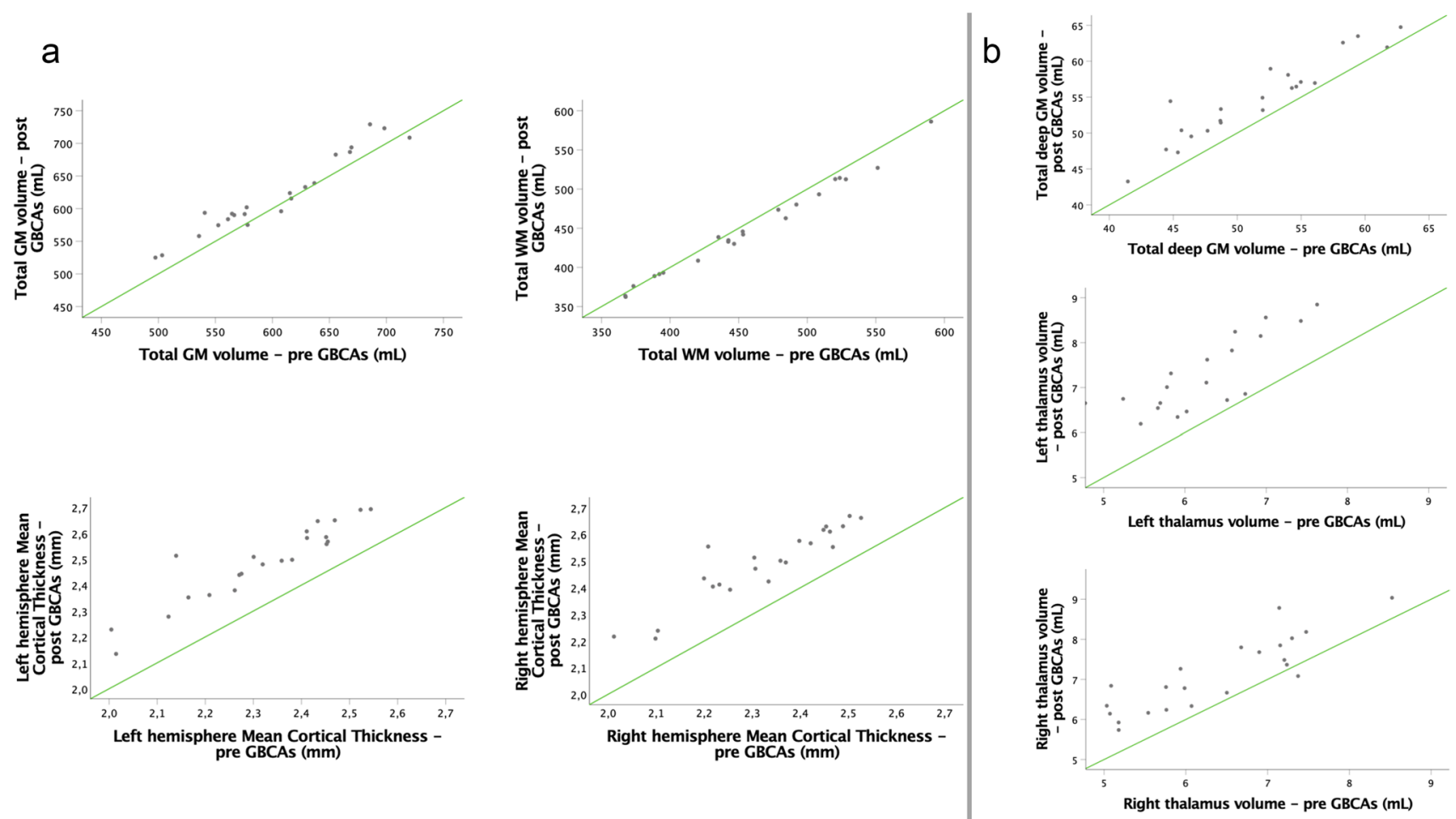

Fig. 5 Scatterplots of global (a) and regional (b) MRI measurements obtained before and after GBCA administration. The green lines indicate identity lines

obtained from post-contrast 3D T1-weighted images. Despite systematic overestimation of the GM, high consistency was observed between all investigated MRI brain measurements obtained before and after administration of GBCAs. a

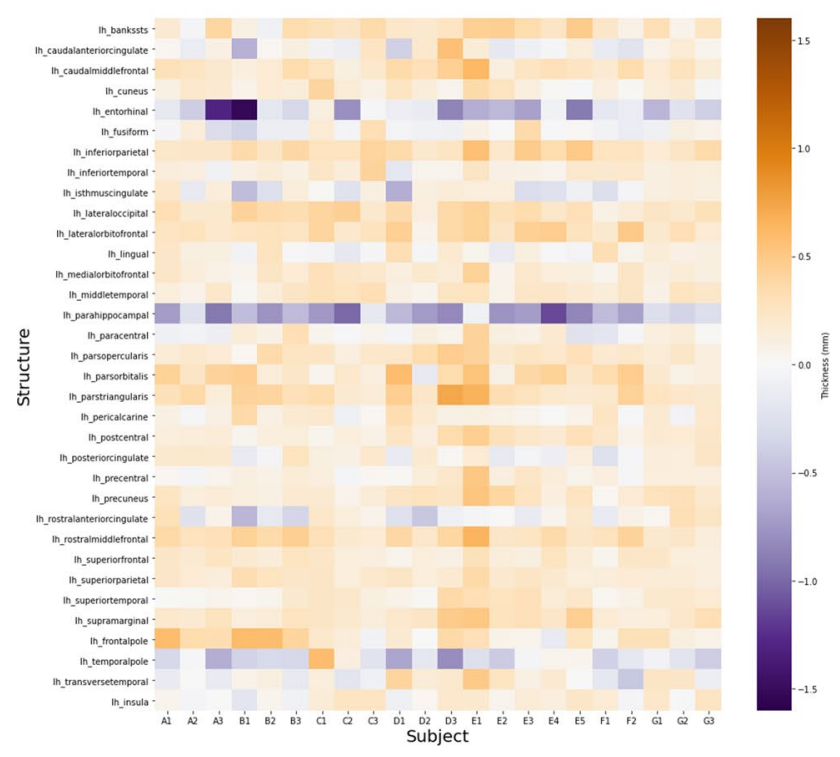

Fig. 6 Heatmaps demonstrating the difference $(\mathrm{mm})$ in cortical thickness in the left (a) and right (b) hemisphere after administration of GBCAs. Brown colours indicate an increase in cortical thickness, and b

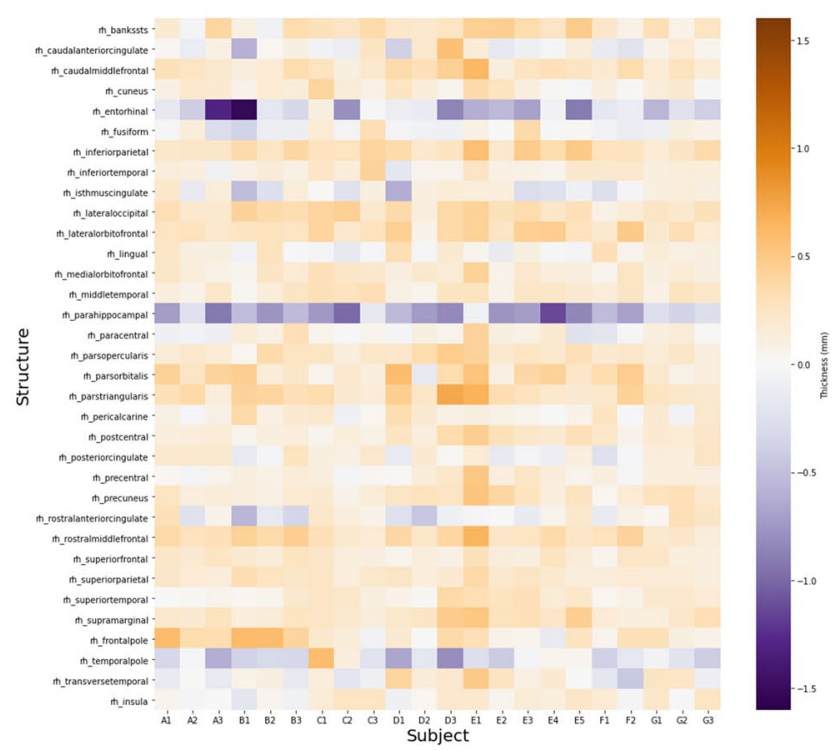

purple colours indicate a decrease in cortical thickness (colour range between $-1.6 \mathrm{~mm}$ and $+1.6 \mathrm{~mm}$ cortical thickness difference). Letters in subject names indicate MRI scanner (a-g) 
Table 5 Image quality metrics obtained by MRI Quality Control Tool

\begin{tabular}{llll}
\hline Image quality metric & $\begin{array}{l}\text { Mean value pre-contrast } \\
(\mathrm{SD})\end{array}$ & $\begin{array}{l}\text { Mean value post-con- } \\
\text { trast (SD) }\end{array}$ & Mean difference $^{\mathrm{a}}(\mathrm{SD})$ \\
\hline CNR & $3.23(0.56)$ & $3.25(0.40)$ & $-0.1(0.52)$ \\
SNR GM & $10.89(2.81)$ & $9.02(2.50)$ & $1.87(2.14)^{*}$ \\
SNR WM & $18.01(3.72)$ & $16.24(3.56)$ & $1.77(1.64)^{* * *}$ \\
CR & $0.39(0.08)$ & $0.45(0.08)$ & $-0.05(0.08)^{*}$ \\
\hline
\end{tabular}

Abbreviations: $S D$, standard deviation; $C N R$, contrast to noise ratio; $S N R$, signal to noise ratio; $G M$, grey matter; $W M$, white matter; $C R$, contrast ratio

${ }^{\text {a }}$ Paired t-test

${ }^{*} p<0.01, * * * p<0.0001$
To our knowledge, this is one of the very few studies investigating the effect of GBCAs on volume measures in MS patients and the first using FreeSurfer. In our study, when investigating the consistency between the measures obtained before and after administration of GBCAs, a good to excellent [35] reliability was found between all investigated measures. This is in agreement with previous studies investigating the whole brain [36], upper cervical cord area [37], and GM and WM measurements [23] using SIENAX $[23,36]$, volBrain, and FSL-Anat [23] and may imply that reliable atrophy measurements acquired from post-contrast images are possible across segmentation techniques.

Consistently, total GM, deep GM, and thalamic volume were between 3.06 and $17.39 \%$ higher in post-contrast images, and the same tendency was found for mean cortical thickness. Simultaneously, total WM volume was $1.74 \%$ lower in postcontrast images. The differences were systematic across all investigated measurements and exhibited no proportional bias. Inspecting cortical segmentations in more detail, we produced heatmaps highlighting within-subject cortical thickness differences in smaller cortical regions (Fig. 6). While smaller regions almost inevitably produce more variability than the larger regions that were the main focus of this work, these inspections showed that cortical thickness overestimation was a brain-wide phenomenon and that the overestimation in postcontrast images was not tied to large errors in any specific region but instead occurred throughout the brain.

These systematic differences in measured volumes and cortical thicknesses between pre- and post-contrast images mean that they should not be compared directly. Another study, using synthetic tissue mapping to measure brain tissue fractions [24], found a $1.1 \%$ increase in total WM fraction and an $0.7 \%$ decrease in GM fraction, in post-contrast images. Due to the methodological differences between that study and ours, it is difficult to assess the reason for the discrepancy in findings.

We could not identify any definite reason for the differences between pre- and post-contrast images. However, when visually inspecting images separately, some recurring soft failures in the FreeSurfer pipeline were found: First, the pial surface often expanded into extraparenchymal tissue, including components of dura or blood vessels as part of the cortex (Fig. 1b). These errors have been shown in areas where the dura or other structures like venous sinuses, lie tangentially in close proximity to the cortex or deep GM structures, leading to larger thickness and volume variability (Fig. 2) [38]. In the FreeSurfer processing stream, the failure to remove enough extraparenchymal tissue happens in the preliminary skull stripping step [39] and the accuracy of the pial surface can be improved by manually erasing the incorporated dura or blood vessels before rerunning analyses [40].

Another recurring soft failure concerned the pial surface. In the surface-based cortical reconstruction, the border between white and grey matter (the white surface) is delineated, following T1 intensity gradients. The pial surface is then grown from the white surface, which serves as a reference point [41]. In all images, but more frequently and severely in post-contrast images, the pial surface failed to follow the white surface, causing "looping” errors (Fig. 1a) and a subsequent incorrect enlargement of the cortical volume and thickness. To improve pial surface accuracy, it is recommended to check for any mistakes in the white surface, and possibly apply manual edits before rerunning analyses [40].

Although most cortical regions demonstrated an increase in cortical thickness in post-contrast images, there were a few exceptions, particularly in the medial part of the temporal lobe. In the entorhinal and parahippocampal gyrus, as well as in the temporal poles, the measured cortical thickness was in some patients thinner after GBCA administration. These regions have in common that they are relatively small and structurally complex, and on visual inspection of the errors, the constructed white surface did not correctly follow the intensity gradients, causing considerable errors in the white surface, and subsequently the pial surface, leaving out parts of the temporal pole (Fig. 3). Challenges in reconstructing parts of the temporal cortex are consistent with previous studies [31, 38, 40, 42], leading to increased variability of the local cortical thickness measurements [38].

The soft failures in the FreeSurfer pipeline occurred more often in post-contrast images in our data. This may be caused by the higher intensity in extraparenchymal structures in close proximity to the cortex or subcortical structures, causing disturbance and challenges in correctly separating different tissue types. 
Skull stripping errors and other soft failures could in some selected regions be identified as the direct cause of increased cortical thickness or GM volume in post-contrast images. It is however uncertain if these errors can explain the systematic increase in almost all GM structures and the overall decrease in WM volume. Even in the absence of active lesions and GBCA leakage through disruptions in the BBB, GBCAs can still be expected to be present in the brain capillary network [24]. This presence may shorten the overall $\mathrm{T} 1$ relaxation time in all tissues, and possibly also affect intensity borders. In our MRIQC analyses, there was no difference between pre- and post-contrast images CNR, indicating that the separation of GM and WM tissue distributions was similar in pre- and post-contrast images. It should however be noted that extracting reliable noise estimates from parallel imaging is challenging.

Systematic effects dependent on the type of GBCA used, dosage, and delay time after administration are likely. In the data retrospectively used in the present study, these factors were not standardised, nor always stated, making them difficult to correct for. To further conclude on the reliability of post-contrast measurements, it is necessary for future research to investigate the possible systematic effects dependent on these variables.

This study is not without limitations. For a multicentre study, the number of patients included was limited, and patients were scanned on different scanners with varying sequence parameters and field strength. Furthermore, some details of the MRI protocol that may affect brain measurements (e.g., head coils $[43,44]$ ) were in some cases neither stated nor retrospectively retrievable, making it difficult to evaluate the effect of these factors. Nonetheless, because the effect of field strength on atrophy measures has been studied before [45], we explored the results for $1.5 \mathrm{~T}$ and $3 \mathrm{~T}$ scanners separately. No systematic differences between the two field strengths regarding the different variabilities in the pre- and postcontrast images emerged, which could be due to small patient numbers and variable acquisition settings. Considering all these aspects, the fact that consistency between measurements before and after GBCA administration was observed across the different scanners, suggests that this behaviour is largely systematic. Future studies should investigate the effect of field strength and those of other aspects of image acquisition more systematically. Image analyses in this study were performed by FreeSurfer, while there are multiple other software packages that have been used in the MS literature. To focus the present work, we chose FreeSurfer because it allows both volumetric and cortical thickness measurements and has been widely used before in MS [46-50].

Finally, we did not perform any pre-processing to remove high-intensity regions (except for those in WM lesions, filled in the lesion filling process) from the post-gadolinium T1-weighted images. Future work should investigate whether removal or replacement of those regions, perhaps similar to the procedure followed as part of the lesion-filling process in the present work, could reduce the observed overestimation of grey matter.

\section{Conclusion}

This study has demonstrated that reliable atrophy measurements may be obtained by FreeSurfer from post-contrast 3D T1-weighted images. A good to excellent consistency was observed between all investigated GM and WM measurements derived from images acquired before and after GBCA administration. However, due to the systematic overestimation of the GM in post-contrast images, measurements acquired from pre- and post-contrast images should not be compared directly, and measurements extracted from certain regions (e.g., the temporal pole) should be interpreted with caution. Furthermore, possible systematic effects dependent on GBCA dose and delay time after injections should be investigated.

Supplementary Information The online version contains supplementary material available at https://doi.org/10.1007/s00330-021-08405-8.

Acknowledgements The authors thank the OFAMS study group and the patients who participated in the study. The authors also thank Joost P.A. Kuijer PhD. from the Department of Radiology and Nuclear Medicine at Amsterdam UMC, MS Center Amsterdam, Amsterdam Neuroscience, Amsterdam UMC, location VUmc, for help with retrieving and interpreting information regarding MRI scanner details.

Funding Open access funding provided by University of Bergen (incl Haukeland University Hospital). K. Wesnes has received funding from Western Norway Regional Health Authority (grant no. \#912020).

The MS Center Amsterdam is funded through a program grant of the Dutch MS Research Foundation (grant no. MS 18-358f).

D.R. van Nederpelt is funded by a joint grant from the Dutch MS Research Foundation and ZonMW (grant no. 446002506) and a grant from Health Holland Life Sciences \& Health (grant no. LSHM19053).

I. Brouwer has been supported by the Dutch MS Research Foundation.

$\varnothing$. Torkildsen and K.M. Myhr are funded by Neuro-SysMed at Haukeland University Hospital and the University of Bergen by a grant from the Research Council of Norway, project number 288164.

F. Barkhof is supported by the National Institute for Health Research (NIHR) biomedical research centre at University College London Hospitals NHS Foundation Trust (UCLH).

\section{Declarations}

Guarantor The scientific guarantor of this publication is Hugo Vrenken, $\mathrm{PhD}$.

Conflict of interest The authors of this manuscript declare no relationships with any companies, whose products or services may be related to the subject matter of the article.

I.A. Lie declares no disclosures relevant to the manuscript.

E. Kerklingh declares no disclosures relevant to the manuscript. 
K. Wesnes has received unrestricted research grants from Novartis and Biogen, and speaker honoraria from Biogen.

D.R. van Nederpelt declares no disclosures relevant to the manuscript. I. Brouwer has received research support from Merck KGaA, Novartis, and Teva.

$\emptyset$. Torkildsen has received research grants and speaker honoraria from Biogen, Roche, Novartis, Merck, and Sanofi.

K.M. Myhr has received unrestricted research grants to his institution; scientific advisory board, and speaker honoraria from Almirall, Biogen, Genzyme, Merck, Novartis, Roche, and Teva; and has participated in clinical trials organised by Biogen, Merck, Novartis, and Roche.

F. Barkhof has received compensation for consulting services and/or speaking activities from Bayer, Biogen Idec, Merck Serono, Novartis, Roche, Teva, Bracco, and IXICO.

$\mathrm{L}$. $\mathrm{B} \emptyset$ has received unrestricted research grants to his institution and/or scientific advisory board or speaker's honoraria from Almirall, Biogen, Genzyme, Merck, Novartis, Roche, and Teva; and has participated in clinical trials organised by Biogen, Merck, Novartis, Roche, and Genzyme.

H. Vrenken has received research grants from Pfizer, Merck Serono, Novartis, and Teva, speaker honoraria from Novartis, and consulting fees from Merck Serono; all funds were paid directly to his institution.

Statistics and biometry No complex statistical methods were used in this paper.

Informed consent Written informed consent was obtained from all subjects (patients) in this study.

Ethical approval The study was approved by the Regional Committee for Medical and Health Research Ethics in Western Norway Regional Health Authority (clinical trials.gov, identifier: NCT00360906).

Study subjects or cohorts overlap Some study subjects or cohorts have been previously reported in " $\omega-3$ fatty acid treatment in multiple sclerosis (OFAMS study): a randomised, double-blind, placebo-controlled trial " by Torkildsen et al., Arch Neurol, 2012. 69(8): p. 1044-51, and in "Low vitamin D, but not tobacco use or high BMI, is associated with long-term disability progression in multiple sclerosis" by Wesnes et al., Mult Scler Relat Disord, 2021. 50: p. 102801.

\section{Methodology \\ - Retrospective \\ - cross-sectional \\ - multicentre study}

Open Access This article is licensed under a Creative Commons Attribution 4.0 International License, which permits use, sharing, adaptation, distribution and reproduction in any medium or format, as long as you give appropriate credit to the original author(s) and the source, provide a link to the Creative Commons licence, and indicate if changes were made. The images or other third party material in this article are included in the article's Creative Commons licence, unless indicated otherwise in a credit line to the material. If material is not included in the article's Creative Commons licence and your intended use is not permitted by statutory regulation or exceeds the permitted use, you will need to obtain permission directly from the copyright holder. To view a copy of this licence, visit http://creativecommons.org/licenses/by/4.0/.

\section{References}

1. Popescu V, Klaver R, Voorn P et al (2015) What drives MRImeasured cortical atrophy in multiple sclerosis? Mult Scler $21: 1280-1290$
2. Horakova D, Dwyer MG, Havrdova E et al (2009) Gray matter atrophy and disability progression in patients with early relapsingremitting multiple sclerosis: a 5-year longitudinal study. J Neurol Sci 282:112-119

3. Zivadinov R, Bergsland N, Dolezal O et al (2013) Evolution of cortical and thalamus atrophy and disability progression in early relapsing-remitting MS during 5 years. AJNR Am J Neuroradiol 34:1931-1939

4. Fisher E, Lee J-C, Nakamura K, Rudick RA (2008) Gray matter atrophy in multiple sclerosis: a longitudinal study. Ann Neurol 64:255-265

5. Sotirchos ES, Gonzalez-Caldito N, Dewey BE et al (2020) Effect of disease-modifying therapies on subcortical gray matter atrophy in multiple sclerosis. Mult Scler 26:312-321

6. Filippi M, Rocca MA, Pagani E et al (2014) Placebo-controlled trial of oral laquinimod in multiple sclerosis: MRI evidence of an effect on brain tissue damage. J Neurol Neurosurg Psychiatry $85: 851-858$

7. Gaetano L, Haring DA, Radue EW et al (2018) Fingolimod effect on gray matter, thalamus, and white matter in patients with multiple sclerosis. Neurology 90:e1324-e1332

8. Kuperberg GR, Broome MR, McGuire PK et al (2003) Regionally localized thinning of the cerebral cortex in schizophrenia. Arch Gen Psychiatry 60:878-888

9. Rosas HD, Liu AK, Hersch S et al (2002) Regional and progressive thinning of the cortical ribbon in Huntington's disease. Neurology 58:695-701

10. Tustison NJ, Cook PA, Klein A et al (2014) Large-scale evaluation of ANTs and FreeSurfer cortical thickness measurements. Neuroimage 99:166-179

11. Schmidt MF, Storrs JM, Freeman KB et al (2018) A comparison of manual tracing and FreeSurfer for estimating hippocampal volume over the adult lifespan. Hum Brain Mapp 39:2500-2513

12. Cardinale F, Chinnici G, Bramerio M et al (2014) Validation of FreeSurfer-estimated brain cortical thickness: comparison with histologic measurements. Neuroinformatics 12:535-542

13. Eshaghi A, Prados F, Brownlee WJ et al (2018) Deep gray matter volume loss drives disability worsening in multiple sclerosis. Ann Neurol 83:210-222

14. Steenwijk MD, Geurts JJ, Daams M et al (2016) Cortical atrophy patterns in multiple sclerosis are non-random and clinically relevant. Brain 139:115-126

15. Calabrese M, Atzori M, Bernardi V et al (2007) Cortical atrophy is relevant in multiple sclerosis at clinical onset. J Neurol 254:1212-1220

16. Zijdenbos AP, Forghani R, Evans AC (2002) Automatic "pipeline" analysis of 3-D MRI data for clinical trials: application to multiple sclerosis. IEEE Trans Med Imaging 21:1280-1291

17. Nakamura K, Fox R, Fisher E (2011) CLADA: cortical longitudinal atrophy detection algorithm. Neuroimage 54:278-289

18. Smith SM, Zhang Y, Jenkinson M et al (2002) Accurate, robust, and automated longitudinal and cross-sectional brain change analysis. Neuroimage 17:479-489

19. Rovira À, Wattjes MP, Tintoré M et al (2015) MAGNIMS consensus guidelines on the use of MRI in multiple sclerosis-clinical implementation in the diagnostic process. Nat Rev Neurol 11:471-482

20. Saade C, Bou-Fakhredin R, Yousem DM, Asmar K, Naffaa L, El-Merhi F (2018) Gadolinium and multiple sclerosis: vessels, barriers of the brain, and glymphatics. AJNR Am J Neuroradiol 39:2168-2176

21. Rogosnitzky M, Branch S (2016) Gadolinium-based contrast agent toxicity: a review of known and proposed mechanisms. Biometals 29:365-376

22. Kanda T, Oba H, Toyoda K, Kitajima K, Furui S (2016) Brain gadolinium deposition after administration of gadolinium-based contrast agents. Jpn J Radiol 34:3-9 
23. Hannoun S, Baalbaki M, Haddad R et al (2018) Gadolinium effect on thalamus and whole brain tissue segmentation. Neuroradiology 60:1167-1173

24. Warntjes JB, Tisell A, Landtblom AM, Lundberg P (2014) Effects of gadolinium contrast agent administration on automatic brain tissue classification of patients with multiple sclerosis. AJNR Am J Neuroradiol 35:1330-1336

25. Torkildsen O, Wergeland S, Bakke S et al (2012) omega-3 fatty acid treatment in multiple sclerosis (OFAMS Study): a randomised, double-blind, placebo-controlled trial. Arch Neurol 69:1044-1051

26. McDonald WI, Compston A, Edan G et al (2001) Recommended diagnostic criteria for multiple sclerosis: guidelines from the International Panel on the diagnosis of multiple sclerosis. Ann Neurol 50:121-127

27. Schmidt P, Gaser C, Arsic M et al (2012) An automated tool for detection of FLAIR-hyperintense white-matter lesions in Multiple Sclerosis. Neuroimage 59:3774-3783

28. Battaglini M, Jenkinson M, De Stefano N (2012) Evaluating and reducing the impact of white matter lesions on brain volume measurements. Hum Brain Mapp 33:2062-2071

29. Fischl B (2012) FreeSurfer Neuroimage 62:774-781

30. Dale AM, Fischl B, Sereno MI (1999) Cortical surface-based analysis. I. Segmentation and surface reconstruction. Neuroimage 9:179-194

31. Desikan RS, Ségonne F, Fischl B et al (2006) An automated labeling system for subdividing the human cerebral cortex on MRI scans into gyral based regions of interest. Neuroimage 31:968-980

32. Esteban O, Birman D, Schaer M, Koyejo OO, Poldrack RA, Gorgolewski KJ (2017) MRIQC: advancing the automatic prediction of image quality in MRI from unseen sites. PLoS One 12:e0184661

33. Zhang Y, Brady M, Smith S (2001) Segmentation of brain MR images through a hidden Markov random field model and the expectation-maximization algorithm. IEEE Trans Med Imaging 20:45-57

34. Giavarina D (2015) Understanding Bland Altman analysis. Biochem Med 25:141-151

35. Koo TK, Li MY (2016) A guideline of selecting and reporting intraclass correlation coefficients for reliability research. J Chiropr Med 15:155-163

36. Neacsu V, Jasperse B, Korteweg T et al (2008) Agreement between different input image types in brain atrophy measurement in multiple sclerosis using SIENAX and SIENA. J Magn Reson Imaging 28:559-565

37. Liu Y, Lukas C, Steenwijk MD et al (2016) Multicenter validation of mean upper cervical cord area measurements from head 3D T1-weighted MR imaging in patients with multiple sclerosis. AJNR Am J Neuroradiol 37:749-754
38. Han X, Jovicich J, Salat D et al (2006) Reliability of MRI-derived measurements of human cerebral cortical thickness: the effects of field strength, scanner upgrade and manufacturer. Neuroimage 32:180-194

39. Fennema-Notestine C, Ozyurt IB, Clark CP et al (2006) Quantitative evaluation of automated skull-stripping methods applied to contemporary and legacy images: effects of diagnosis, bias correction, and slice location. Hum Brain Mapp 27:99-113

40. McCarthy CS, Ramprashad A, Thompson C, Botti J-A, Coman IL, Kates WR (2015) A comparison of FreeSurfer-generated data with and without manual intervention. Front Neurosci. https://doi. org/10.3389/fnins.2015.00379

41. Fischl B, Dale AM (2000) Measuring the thickness of the human cerebral cortex from magnetic resonance images. Proc Natl Acad Sci USA 97:11050-11055

42. Oguz I, Cates J, Fletcher T et al (2008) Cortical correspondence using entropy-based particle systems and local features.https:// doi.org/10.1109/ISBI.2008.4541327

43. Panman JL, To YY, van der Ende EL et al (2019) Bias introduced by multiple head coils in MRI research: an 8 channel and 32 channel coil comparison. Front Neurosci. https://doi.org/10.3389/fnins.2019.00729

44. Li H, Smith SM, Gruber S et al (2018) Combining multi-site/ multi-study MRI data: linked-ICA denoising for removing scanner and site variability from multimodal MRI data. bioRxiv. https:// doi.org/10.1101/337576:337576

45. Battaglini M, Gentile G, Luchetti L et al (2019) Lifespan normative data on rates of brain volume changes. Neurobiol Aging 81:30-37

46. Guo C, Ferreira D, Fink K, Westman E, Granberg T (2019) Repeatability and reproducibility of FreeSurfer, FSL-SIENAX and SPM brain volumetric measurements and the effect of lesion filling in multiple sclerosis. Eur Radiol 29:1355-1364

47. Platten M, Martola J, Fink K, Ouellette R, Piehl F, Granberg T (2020) MRI-based manual versus automated corpus callosum volumetric measurements in multiple sclerosis. J Neuroimaging 30:198-204

48. Calabrese M, Rinaldi F, Grossi P, Gallo P (2011) Cortical pathology and cognitive impairment in multiple sclerosis. Expert Rev Neurother 11:425-432

49. Inglese M, Petracca M, Mormina E et al (2017) Cerebellar volume as imaging outcome in progressive multiple sclerosis. PLoS One 12:e0176519

50. Chu R, Kim G, Tauhid S, Khalid F, Healy BC, Bakshi R (2018) Whole brain and deep gray matter atrophy detection over 5 years with 3T MRI in multiple sclerosis using a variety of automated segmentation pipelines. PLoS One 13:e0206939

Publisher's note Springer Nature remains neutral with regard to jurisdictional claims in published maps and institutional affiliations. 